\title{
Catarata transitoria y atrofia permanente del iris posterior a la aplicación de 5-fluorouracilo subconjuntival
}

\author{
Transient cataract and permanent iris atrophy after \\ subconjunctival 5-fluorouracil injection
}

\author{
Jasbeth Ledesma Gil," Lulu Ululani Gurría Quintana*
}

Citar como: Ledesma GJ, Gurría QLU. Catarata transitoria y atrofia permanente del iris posterior a la aplicación de 5-fluorouracilo subconjuntival. An Med (Mex). 2020; 65 (4): 301-304. https://dx.doi.org/10.35366/97468

\section{RESUMEN}

La entrada inadvertida de 5-FU a la cámara anterior durante una aplicación subconjuntival podría causar catarata transitoria y atrofia permanente de iris, por lo que se recomienda su uso cauteloso en pacientes fáquicos. El objetivo es reportar un caso de catarata transitoria y atrofia permanente de iris posterior a revisión de ampolla filtrante y aplicación subconjuntival de 5-fluorouracilo. Reporte de caso: paciente femenino de 49 años con ampolla filtrante encapsulada posterior a cirugía de trabeculectomía. Se realizó revisión de ampolla filtrante con inyección de $5 \mathrm{mg}$ de 5 -fluorouracilo adyacente a la ampolla filtrante. Inmediatamente posterior al procedimiento la paciente refiere baja visual debido a la opacificación de la cápsula anterior del cristalino. Seis semanas después, la agudeza visual mejoró, pero la atrofia de iris persistió. La agudeza visual a los seis meses fue de 20/25. En conclusión, se puede presentar formación de catarata transitoria debido a la inyección inadvertida de 5-FU en la cámara anterior.

Palabras clave: Glaucoma, catarata, 5-fluorouracilo.

Nivel de evidencia: IV

\section{ABSTRACT}

Accidental 5-FU into the anterior chamber after subconjunctival injection could produce transient cataract and iris atrophy, it is important to be careful in phakic patients. The main purpose of this report is to describe a case of transient cataract formation and iris atrophy after bled needling with 5-FU. Case report: a 49-years-old female underwent subconjunctival injection of $5 \mathrm{mg} 5$-fluorouracil after trabeculectomy. Immediately after injection, she presented decrease visual acuity secondary to crystalline lens opacity, after six weeks visual acuity improved nevertheless iris atrophy remained. Visual acuity six months after was 20/25. In conclusion, transient cataract formation and iris atrophy can occur after accidental injection of 5-FU into the anterior chamber.

Keywords: Glaucoma, cataract, 5-fluorouracil.

Level of evidence: $I V$

\footnotetext{
* Departamento de Glaucoma. Instituto de Oftalmología Fundación Conde de Valenciana ABC de Santa Fe. Ciudad de México, México.
}

\section{Recibido para publicación: 03/11/2020. Aceptado: 10/11/2020.}

Abreviaturas:

Needling = revisión de ampolla filtrante.

$$
5 \text {-FU = 5-fluorouracilo. }
$$

\section{Correspondencia:}

Jasbeth Ledesma Gil

Prolongación Vasco de Quiroga Núm. 4001, Torre A, 5to piso,

Consultorio 5, Santa Fe, 05310, Cuajimalpa,

Ciudad de México, México.

Tel: 55 9152-2030

E-mail: jasbeth.ledesma@institutodeoftalmologia.org 


\section{INTRODUCCIÓN}

La trabeculectomía continúa siendo el estándar de oro de la cirugía de glaucoma para los pacientes en quienes ha fallado la terapia médica máxima tolerada. ${ }^{1}$ La falla de la ampolla filtrante subconjuntival es un problema serio y frecuente dentro de la evolución de la cirugía filtrante. La poca filtración se debe comúnmente al desarrollo de fibrosis que involucra la interfase epiesclera-cápsula de Tenon-conjuntiva. Diversos estudios han demostrado la utilidad de sustancias como el 5-fluorouracilo (5-FU) y la mitomicina $\mathrm{C}$ durante y después de la cirugía para reducir la proliferación de fibroblastos y modular la cicatrización, mejorando el éxito de la cirugía filtrante. ${ }^{2}$ Se han propuesto diferentes enfoques para manejar las ampollas filtrantes que dejan de funcionar apropiadamente y la revisión con aguja de las ampollas filtrantes fallidas (needling) ha sido una alternativa útil, relativamente simple y segura para manejar esos casos. ${ }^{3}$ Las aplicaciones subconjuntivales postoperatorias de 5-FU mejoran el éxito de la cirugía filtrante en glaucoma. ${ }^{4,5}$ A continuación se presenta un caso con catarata transitoria posterior a la inyección subconjuntival de 5-FU.

\section{CASO CLÍNICO}

Paciente femenino de 49 años, con diagnóstico de glaucoma juvenil con daño avanzado en ambos ojos (Figura 1). Antecedente de cirugía de trabeculectomía con antimetabolito (mitomicina $\mathrm{C}$ ) en ambos

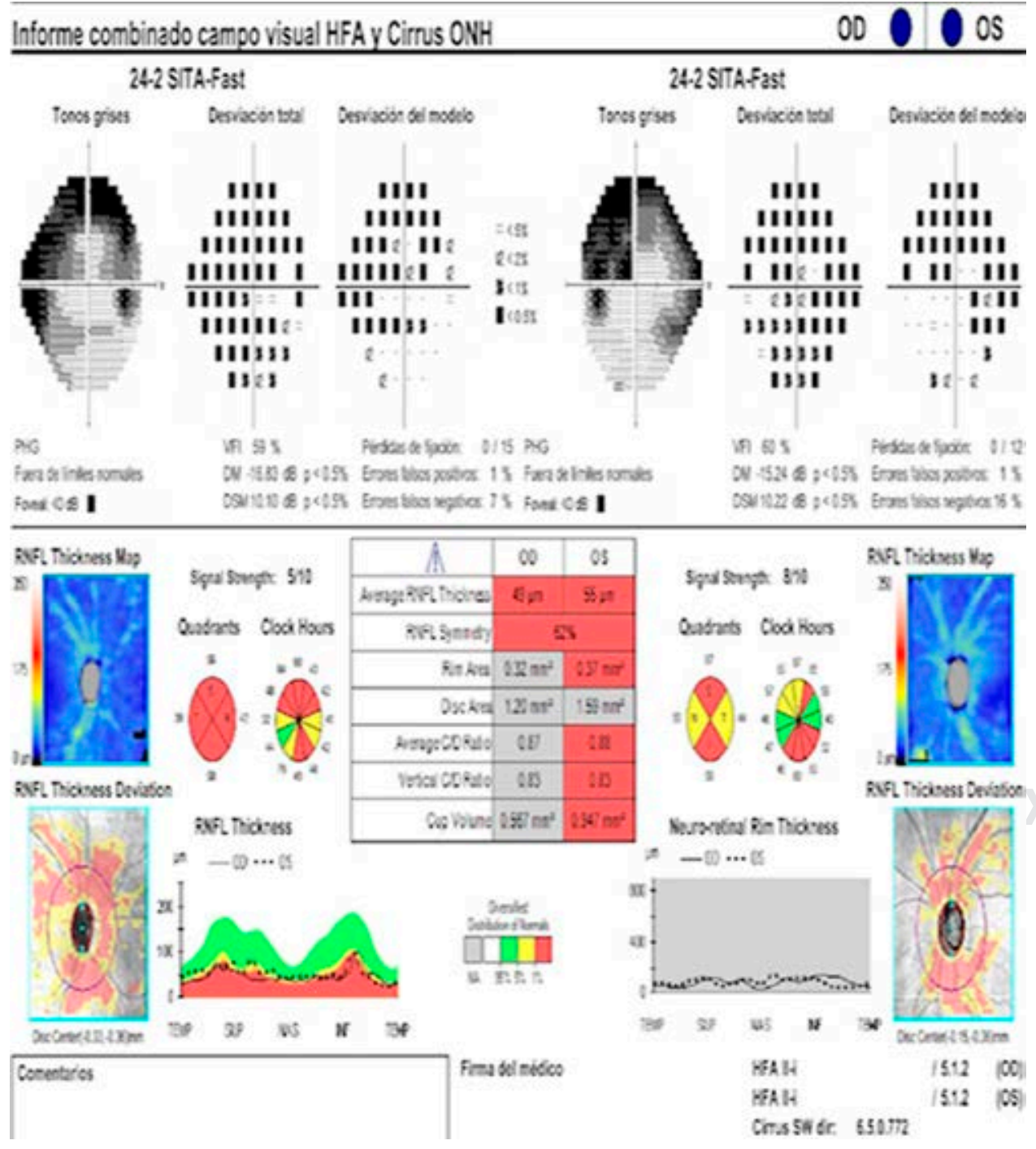

Figura 1:

Campo visual y tomografía de coherencia óptica de nervio óptico con daño glaucomatoso severo en ambos ojos. 


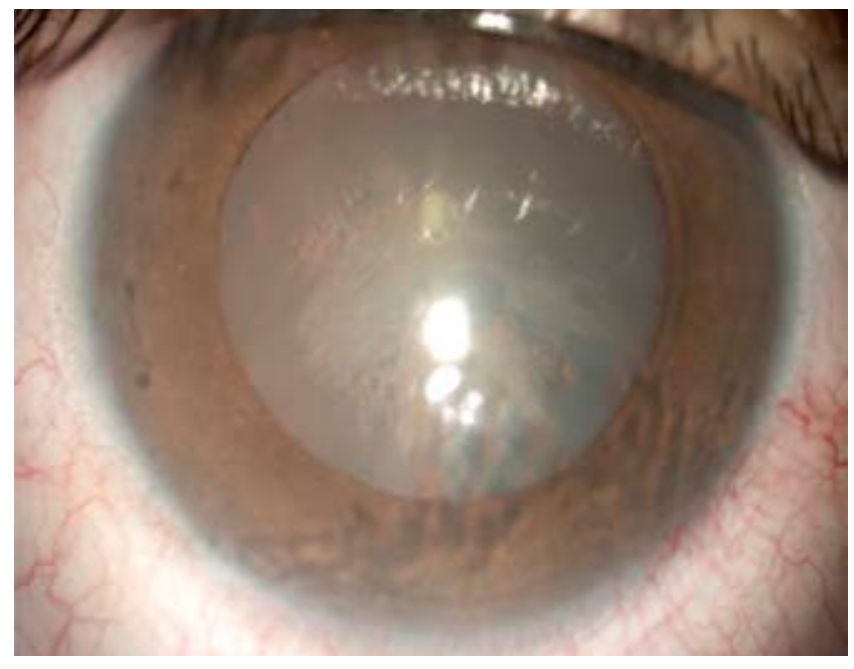

Figura 2: Catarata subcapsular anterior central después de la aplicación subconjuntival de 5-fluorouracilo.

ojos. Acude a revisión de rutina encontrándose a la exploración oftalmológica agudeza visual de 20/25 en ambos ojos, presión intraocular de $28 \mathrm{mmHg}$ en ojo derecho y de $12 \mathrm{mmHg}$ en ojo izquierdo con relación copa disco de 8/10 en ambos ojos. En ojo derecho se observa una bula quística elevada focalmente, circunscrita sin flujo posterior, por tal motivo se decide realizar needling en ojo derecho con aplicación subconjuntival de 5-FU. Previa colocación de gotas de clorhidrato de tetracaína al 0.5\%, aplicación en fondo de saco de solución de yodopovidona y aseo periocular con yodopovidona. Se realiza needling con aguja calibre 27 en el borde temporal de la bula, se confirma el restablecimiento del drenaje del humor acuoso al observar un aumento en el volumen de la ampolla y se inyecta $0.2 \mathrm{~mL} 2.5 \%$ (5 mg) de 5-FU subconjuntival $5 \mathrm{~mm}$ temporal al sitio de la ampolla. Inmediatamente después de la aplicación del 5-FU, la paciente refirió disminución súbita de la visión. La agudeza visual que se encontraba previamente en 20/25 disminuyó a movimientos de manos, se aprecia presencia de midriasis y catarata cortical anterior central (Figura 2). Se indicó manejo con acetato de prednisolona cada dos horas y moxifloxacino cada cuatro horas. 72 horas después, la agudeza visual mejoró a 20/60 en ojo derecho con reducción significativa de la opacidad cortical anterior central, pero con presencia de atrofia de iris inferior. A las seis semanas la agudeza visual regresó a 20/25, pero persistió la atrofia de iris (Figura 3). La microscopia especular mostró un adecuado conteo celular en ambos ojos (Figura 4).

\section{DISCUSIÓN}

La entrada inadvertida de 5-FU a la cámara anterior durante una aplicación subconjuntival podría causar catarata transitoria y atrofia permanente de iris, por lo que se recomienda su uso cauteloso en pacientes fáquicos. Existe un reporte previo de catarata transitoria posterior a needling e inyección subconjuntival de 5-FU en un paciente de 51 años de edad, el cual es similar a nuestro reporte de caso, cursó con disminución significativa de la agudeza visual (cuenta dedos a $30 \mathrm{~cm}$ ) y opacidad de la cápsula anterior en los cinco minutos posteriores a la aplicación del medicamento, con resolución completa de la opacidad de la cápsula anterior y regreso a la agudeza visual previa (20/30) al mes del procedimiento. ${ }^{6}$ Robert F. Rothman y colaboradores ${ }^{7}$ reportaron un caso de queratopatía cristalina no infecciosa posterior a la aplicación subconjuntival de 5-FU en una paciente operada de trabeculectomía, la cual remitió con el uso de esteroides tópicos. En nuestro caso la agudeza visual disminuyó inmediatamente y regresó de manera paulatina al estado previo al procedimiento a las seis semanas, razón por la que recomendamos manejo con antiinflamatorio esteroideo y vigilancia antes de decidir cirugía de facoemulsificación de cristalino debido a la posibilidad de recuperación a través del tiempo. Además, nuestra paciente desarrolló atrofia de iris permanente, lo cual no representa ningún riesgo para la función o visión, y puede deberse a una mayor entrada intracameral de 5 -FU en

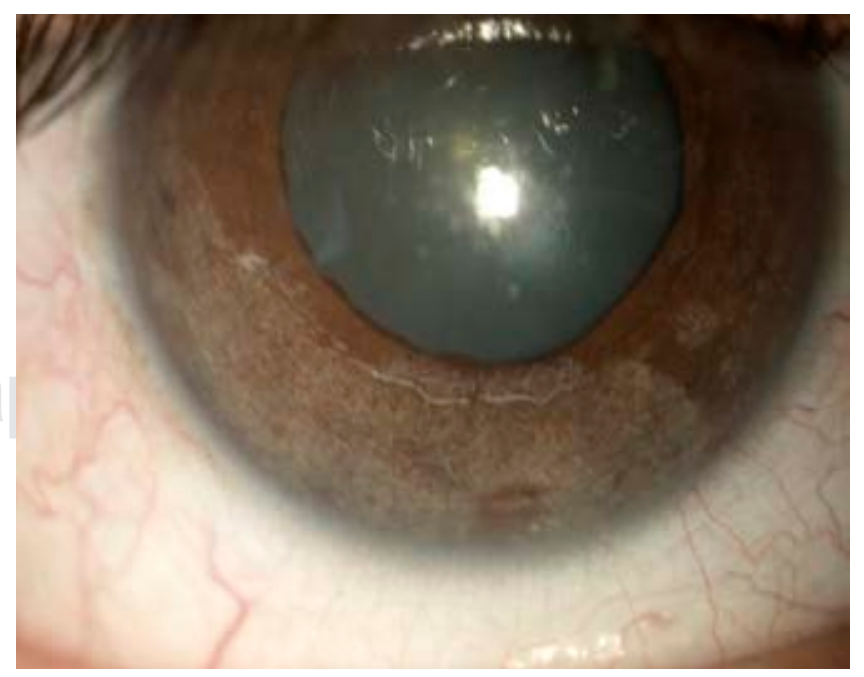

Figura 3: Recuperación de la transparencia del cristalino seis semanas posteriores a la aplicación de 5-fluorouracilo. Presencia de atrofia sectorial del iris en región inferior. 

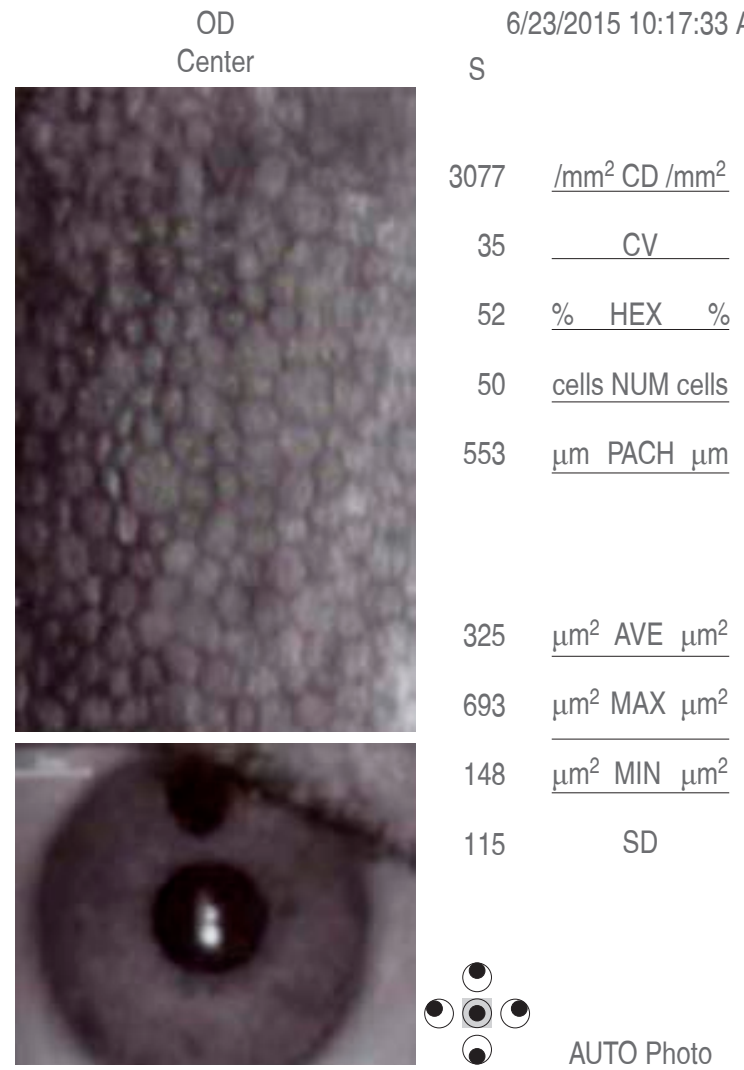

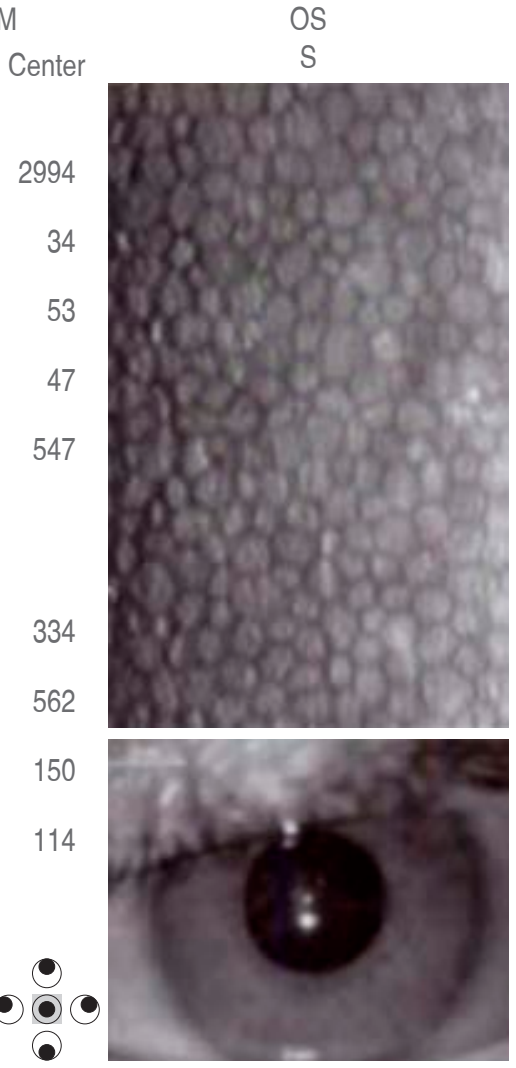

Figura 4:

Microscopia especular que muestra adecuado conteo endotelial en ambos ojos. la cámara anterior. La opacidad de la cápsula anterior probablemente es producida por la actividad que ejerce el 5-FU sobre el metabolismo del cristalino y que esto sea un evento dosis dependiente, ya que la aplicación de 5-FU subconjuntival se ha utilizado de manera segura durante décadas para el control de la fibrosis conjuntival en un gran número pacientes fáquicos con cirugía de trabeculectomía sin presentarse efectos adversos o secundarios no deseados. ${ }^{4}$

\section{CONCLUSIÓN}

Se puede presentar formación de catarata transitoria debido a la inyección inadvertida de 5-FU en la cámara anterior, por lo que los médicos oftalmólogos debemos estar atentos a esta complicación y esperar su resolución antes de proponer manejo quirúrgico a los pacientes.

\section{BIBLIOGRAFÍA}

1. Fagerli M, Løfors KT, Elsås T. Needling revision of failed filtering blebs after trabeculectomy: a retrospective study. Acta Ophthalmol Scand [Internet]. 2003 [cited 2020 Aug 17];
81 (6): 577-582. Available from: https://pubmed.ncbi.nlm.nih. gov/14641257/

2. Shetty RK, Wartluft L, Moster MR. Slit-lamp needle revision of failed filtering blebs using high-dose mitomycin C. J Glaucoma [Internet]. 2005 [cited 2020 Aug 17]; 14 (1): 52-56. Available from: http://journals.lww.com/00061198-20050200000010

3. Rockwood EJ, Parrish RK, Heuer DK, Skuta GL, Hodapp E, Palmberg PF et al. Glaucoma filtering surgery with 5-fluorouracil. Ophthalmology. 1987; 94 (9): 1071-1078.

4. Arnljots TS, Kasina R, Bykov VJN, Economou MA. Needling with 5-fluorouracil (5-fu) after xen gel stent implantation: 6-month outcomes. J Glaucoma [Internet]. 2018 [cited 2020 Aug 17]; 27 (10): 893-899. Available from: http://journals.lww. com/00061198-201810000-00010

5. Zheng L, Arvind H, Wechsler D. Outcomes: trabeculectomy bleb needle revision with 5-fluorouracil. J Glaucoma [Internet]. 2016 [cited 2020 Aug 17]; 25 (3): 317-323. Available from: http://journals.lww.com/00061198201603000-00014

6. Libre PE. Transient, profound cataract associated with intracameral 5-fluorouracil. Am J Ophthalmol [Internet]. 2003 [cited 2020 Aug 17]; 135 (1): 101-102. Available from: https://pubmed.ncbi.nlm.nih.gov/12504710/

7. Rothman RF, Liebmann JM, Ritch R. Noninfectious crystalline keratopathy after postoperative subconjunctival 5-fluorouracil. Am J Ophthalmol [Internet]. 1999 [cited 2020 Aug 17]; 128 (2): 236-237. Available from: https://inkinghub. elsevier.com/retrieve/pii/S0002939499001154 